\title{
Synthesis of dipyrromethanes in aqueous media using boric acid
}

\author{
Anchal Singhal, ${ }^{a}$ Snigdha $\operatorname{Singh}^{b}$ and Shive M. S. Chauhan*a \\ ${ }^{a}$ Department of Chemistry, University of Delhi, Delhi 110 007, India \\ ${ }^{b}$ Department of Chemistry, Hans Raj College, University of Delhi, Delhi 110 007, India \\ E-mail: smschauhanchemistry@gmail.com
}

\section{DOI: https://doi.org/10.24820/ark.5550190.p009.847}

\begin{abstract}
Boric acid acts as an efficient and green catalyst for the condensation reaction of pyrrole and aldehydes in aqueous medium to produce parallel library of dipyrromethanes including new compound analogue, meso-acetyldipyrromethane in moderate to good yield. The use of boric acid as catalyst and selective reactivity of aldehydes over ketones in its presence, and water as reaction medium are important attributes in the present protocol.
\end{abstract}

Keywords: Pyrrole, aldehydes, boric acid, dipyrromethanes, meso-acetyldipyrromethane

\section{Introduction}

Dipyrromethanes are the important precursors for synthesis of various natural and functional porphyrins, porphyrinoid macrocycles, linear porphyrin arrays and expanded porphyrins. ${ }^{1-4}$ Recently, its applications have been extended for synthesis of gold nanoparticles. ${ }^{5}$ The condensation of aldehydes with excess of pyrrole in the presence of acid such as trifluoroacetic acid, ${ }^{6} \mathrm{BF}_{3}$-etherate, ${ }^{7,8} p$-toluenesulfonic acid, ${ }^{9}$ acetic acid, ${ }^{10,11}$ indium chloride, ${ }^{12}$ or metal triflates ${ }^{13}$ in organic solvents or in the absence of solvent has been reported as general methods for synthesis of meso-substituted dipyrromethanes along with oligomeric by-products. ${ }^{14,15}$ The major disadvantages of these methods include the requirement of large amounts of pyrrole, corrosive acidic catalyst and the use of chlorinated solvents. Hence, a mild route to synthesis of dipyrromethanes is desirable due to its vast applications in organic syntheses.

Organic reactions in aqueous media comprise an important area of current research interests including green chemistry. ${ }^{16-18}$ Our research group has developed a few green methods for synthesis of dipyrromethanes and related compounds ${ }^{19-22}$ in addition to other groups. ${ }^{23-25}$ Boric acid has been used as catalyst for synthesis of bis(indolyl)methanes, ${ }^{26}$ benzoxanthenes, ${ }^{27}$ dehydration of glucose, ${ }^{28}$ protection and deprotection of alcohols and ethers, ${ }^{29} \mathrm{~N}$-acylation, ${ }^{30}$ bromination, ${ }^{31}$ aza Michael reactions, ${ }^{32}$ Biginelli reactions ${ }^{33}$ and other condensation reactions. ${ }^{34-36}$ Herein, we have described the use of boric acid as an innocuous and highly efficient catalyst for 
the condensation reaction of pyrrole and aldehydes in aqueous medium to afford the corresponding dipyrromethanes. This investigation presents the simplest protocol for this type of reactions and anticipates as interesting for wide range of organic chemists.

\section{Results and Discussion}

In this report, we describe an efficient method for synthesis of dipyrromethanes using boric acid in aqueous medium at $30^{\circ} \mathrm{C}$ ( $\sim$ room temperature) as depicted in Scheme 1 . The reaction of $37 \%$ formaldehyde with 2.5 equiv. of pyrrole in aqueous solution of boric acid at $30^{\circ} \mathrm{C}$ for $6 \mathrm{~h}$ gave dipyrromethane (3a) in $65 \%$ yield. The reaction of benzaldehyde with pyrrole under similar reaction conditions for $8 \mathrm{~h}$ gave meso-phenyldipyrromethane (3b) in 59\% yield. No product was formed in the absence of boric acid. Further, the reaction of pyrrole with different aldehydes in presence of boric acid in aqueous media was performed under similar reaction conditions to produce a parallel library of dipyrromethanes.

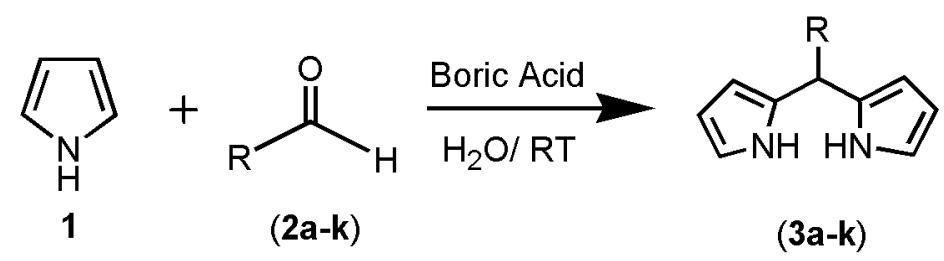

Scheme 1. Key reaction for synthesis of dipyrromethanes.

The reaction of ketones with pyrrole catalyzed by boric acid in aqueous solution at $30^{\circ} \mathrm{C}$ did not provide any product. This was further confirmed when the reaction of methylglyoxal with pyrrole gave only meso-acetyl dipyrromethane (3k) under similar conditions for $12 \mathrm{~h}$. (This compound is introduced for the first time and has never been reported in the literature to the best of our knowledge). This confirmed that aldehyde was reacted selectively with pyrrole in aqueous solution of boric acid. This could be attributed to low reactivity of ketones as compared to aldehydes and mild nature of boric acid. The reaction time and yield of different dipyrromethanes are summarized in Table 1.

Its easy availability, cost effectiveness and environmental compatibility in addition to weak acidity and ability to produce $\mathrm{H}^{+}$from its reaction with water, $\mathrm{B}(\mathrm{OH})_{3}+\mathrm{H}_{2} \mathrm{O} \rightarrow \mathrm{B}(\mathrm{OH})_{4}{ }^{-}+\mathrm{H}^{+}$ were some of the most important reasons for selecting boric acid as catalyst. Further, because of easy handling and operation, its weak acidity lead to the desired product selectively in high efficacy without any side products. 
Table 1 Dipyrromethane derivatives $3 \mathbf{a}-\mathbf{k}$ produced according to Scheme 1

\begin{tabular}{cccc}
\hline Product & $\mathrm{R}$ & Time (h) & Yield (\%) \\
\hline 3a & $\mathrm{H}$ & 6 & 65 \\
$\mathbf{3 b}$ & $\mathrm{Ph}$ & 8 & 59 \\
$\mathbf{3 c}$ & $4-\mathrm{ClC}_{6} \mathrm{H}_{4}$ & 12 & 65 \\
$\mathbf{3 d}$ & $4-\mathrm{FC}_{6} \mathrm{H}_{4}$ & 12 & 63 \\
$\mathbf{3 e}$ & $4-\mathrm{BrC}_{6} \mathrm{H}_{4}$ & 12 & 65 \\
$\mathbf{3 f}$ & $4-\mathrm{O}_{2} \mathrm{NC}_{6} \mathrm{H}_{4}$ & 12 & 63 \\
$\mathbf{3 g}$ & $4-\mathrm{CH}_{3} \mathrm{C}_{6} \mathrm{H}_{4}$ & 10 & 68 \\
$3 \mathbf{3 h}$ & $2,4,6-\left(\mathrm{CH}_{3}\right)_{3} \mathrm{C}_{6} \mathrm{H}_{2}$ & 10 & 67 \\
$3 \mathbf{i}$ & $4-\mathrm{CH}_{3} \mathrm{OC}_{6} \mathrm{H}_{4}$ & 10 & 69 \\
$\mathbf{J}$ & $4-\mathrm{pyridyl}$ & 12 & 68 \\
$\mathbf{3 k}$ & $\mathrm{CH}_{3} \mathrm{CO}$ & 12 & 70 \\
\hline
\end{tabular}

The condensation reaction of pyrrole with aldehydes is believed to occur at the interface of organic and aqueous layers. The release of dipyrromethane from the aqueous layer through interphase to organic layer was responsible for completion of the reaction and protection of the dipyrromethane from further reactions. The prolonging of the reaction time and changing the reaction conditions lead to the formation of some undesirable products such as tripyrromethane and other higher oligomers as observed by TLC. The reaction of pyrrole with aldehydes in aqueous boric acid solution gave the pure dipyrromethanes in high yields (Table 1).

The plausible mechanism of the reaction could be explained as that reaction proceeded with protonation of carbonyl oxygen of aldehyde molecule in presence of aqueous solution of boric acid followed by nucleophilic attack at the $\alpha$-position of pyrrole. Deprotonation at this step stabilized the intermediate. Further protonation and subsequent reaction with a second molecule of pyrrole with the loss of water molecule followed by deprotonation gives dipyrromethane (Scheme 2). 


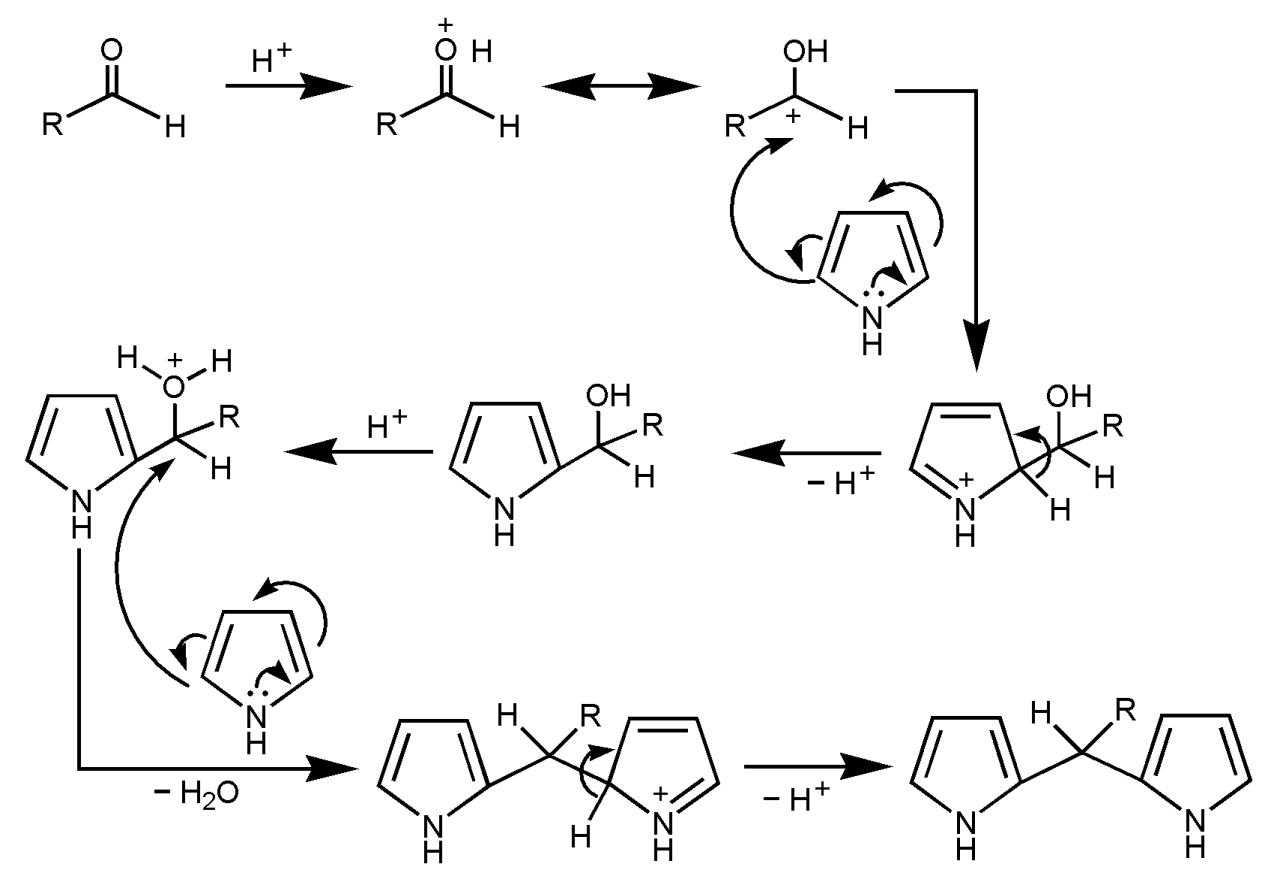

Scheme 2. Plausible mechanism for the synthesis of dipyrromethane.

\section{Conclusions}

Boric acid is advocated as mild and eco-friendly catalyst for the synthesis of a parallel library of dipyrromethanes including new compound analogue, meso-acetyldipyrromethane in aqueous medium. Boric acid, being mild in nature, offered desired dipyrromethanes selectively without any polymerization of the products or other side reactions. The advantages of the present method include avoiding the use of large excess of pyrrole and corrosive acidic catalyst.

\section{Experimental Section}

General Information. All chemicals used were of analytical grade. Pyrrole, aldehydes and solvents were freshly distilled prior to use. Identities of all known products were confirmed by comparison with their physical and spectral data. The dipyrromethanes were easily visualized upon exposure of thin layer chromatography plates to iodine vapor. ${ }^{1} \mathrm{H}$ NMR spectra were recorded on a Bruker Avance Spectrospin $300 \mathrm{MHz}$ spectrophotometer. The chemical shifts in parts per million (ppm) for proton $\left({ }^{1} \mathrm{H}\right)$ NMR spectra were internally referenced relative to tetramethylsilane (TMS, 
$0.00 \mathrm{ppm}$ ). The mass spectra (ESI-MS) were recorded on Micromass LCT KC-455 instrument using electron spray positive ion mode.

\section{General reaction procedure for the synthesis of dipyrromethanes}

Aldehyde (20 mmol, 1 equiv.) was added to aqueous solution of boric acid (248 $\mathrm{mg}$ in $10 \mathrm{ml}$ of water) followed by dropwise addition of pyrrole $(3.35 \mathrm{~g}, 50 \mathrm{mmol})$. The reaction mixture was stirred on a magnetic stirrer in an open atmosphere at $30^{\circ} \mathrm{C}$ and the progress of the reaction was monitored by TLC. After completion of the reaction (when aldehyde was consumed, as checked by TLC), the organic layer was separated and aqueous layer was extracted with dichloromethane, the combined organic layers were dried over anhydrous $\mathrm{Na}_{2} \mathrm{SO}_{4}$ and purified by column chromatography over silica gel (60-120 mesh) using petroleum ether-chloroform (ratio varied with product) to give corresponding dipyrromethanes respectively. The spectral data of the compounds are reported below:

Dipyrromethane (3a). Greyish solid, mp $73{ }^{\circ} \mathrm{C}$ (lit. ${ }^{11} \mathrm{mp} 75^{\circ} \mathrm{C}$, lit. yield $\left.{ }^{11} 41 \%\right) .{ }^{1} \mathrm{H}$ NMR $(300$ $\left.\mathrm{MHz}, \mathrm{CDCl}_{3}\right): 3.92$ (s, 2H), 6.02 (s, 2H), 6.14 (d, J $\left.2.4 \mathrm{~Hz}, 2 \mathrm{H}\right), 6.64$ (s, 2H), 7.83 (s, 2H, NH). MS $m / z(\%): 146[\mathrm{M}]^{+}$(100) calc. for $\mathrm{C}_{9} \mathrm{H}_{10} \mathrm{~N}_{2}$.

meso-Phenyldipyrromethane (3b). White crystals, mp $100{ }^{\circ} \mathrm{C}$ (lit. ${ }^{11} \mathrm{mp} \mathrm{100-101}{ }^{\circ} \mathrm{C}$, lit. yield ${ }^{11}$ 53\%). ${ }^{1} \mathrm{H}$ NMR (300 MHz, $\left.\mathrm{CDCl}_{3}\right): 5.48$ (s, 1H), 5.92 (s, 2H), 6.16 (q, 2H), 6.70 (d, 2H), 7.217.32 (m, 5H), 7.92 (brs, 2H). MS m/z (\%): $222\left(\mathrm{M}^{+}, 97\right),\left(\mathrm{M}^{+}-\mathrm{C}_{6} \mathrm{H}_{5}, 100\right)$ calc. for $\mathrm{C}_{15} \mathrm{H}_{14} \mathrm{~N}_{2}$.

meso-Chlorophenyldipyrromethane (3c). Pale yellow solid, mp $110{ }^{\circ} \mathrm{C}$ (lit. ${ }^{13} \mathrm{mp} 112-113{ }^{\circ} \mathrm{C}$, lit. yield $\left.{ }^{13} 44 \%\right)$. ${ }^{1} \mathrm{H}$ NMR (300 MHz, $\left.\mathrm{CDCl}_{3}\right)$ : 5.44 (s, 1H), $5.88(\mathrm{~m}, 2 \mathrm{H}), 6.24(\mathrm{~m}, 2 \mathrm{H}), 6.70$ (m, 2H), 7.24-7.29 (m, 2H), 7.93 (brs, 2H, NH). MS m/z (\%): $\left.256\left(\mathrm{M}^{+}, 43\right), 258(\mathrm{M}+2)^{+}, 14\right), 145\left(\mathrm{M}^{+}-\right.$ $\mathrm{C}_{6} \mathrm{H}_{4} \mathrm{Cl}, 100$ ) calc. for $\mathrm{C}_{15} \mathrm{H}_{13} \mathrm{ClN}_{2}$.

meso-Fluorophenyldipyrromethane (3d). White crystals, mp $81{ }^{\circ} \mathrm{C}$ (lit. ${ }^{11} \mathrm{mp} 81{ }^{\circ} \mathrm{C}$, lit. yield ${ }^{11}$ 28\%). ${ }^{1} \mathrm{H}$ NMR (300 MHz, $\left.\mathrm{CDCl}_{3}\right): 5.49$ (s, $\left.1 \mathrm{H}\right), 5.93(\mathrm{~m}, 2 \mathrm{H}), 6.20$ (dd, J 2.8 Hz, 2H), 6.74 (m, 2H), 7.04 (m, 2H), $7.94(\mathrm{~m}, 2 \mathrm{H}), 8.18$ (brs, 2H, NH). MS m/z (\%): $240\left(\mathrm{M}^{+}, 100\right), 145\left(\mathrm{M}^{+}-\mathrm{C}_{6} \mathrm{H}_{4} \mathrm{~F}\right.$, 45) calculated for $\mathrm{C}_{15} \mathrm{H}_{13} \mathrm{FN}_{2}$.

meso-Bromophenyldipyrromethane (3e). White solid, mp $122{ }^{\circ} \mathrm{C}$ (lit. ${ }^{13} \mathrm{mp} 122-123{ }^{\circ} \mathrm{C}$, lit. yield ${ }^{13} 20 \%$ ). ${ }^{1} \mathrm{H}$ NMR (300 MHz, $\left.\mathrm{CDCl}_{3}\right): 5.43$ (s, 1H), $5.86(\mathrm{~s}, 2 \mathrm{H}), 6.15$ (dd, J 4.5 Hz, 2H), 6.68 (d, J 8.2 Hz, 2H), 7.10-7.24 (m, 4H), 7.92 (brs, 2H, NH). MS m/z (\%): $301\left(\mathrm{M}^{+}, 100\right), 303\left[(\mathrm{M}+2)^{+}\right.$, 98], $112\left(\mathrm{M}^{+}-\mathrm{C}_{6} \mathrm{H}_{4} \mathrm{Br}, 80\right)$ calculated for $\mathrm{C}_{15} \mathrm{H}_{13} \mathrm{BrN}_{2}$.

meso-Nitrophenyldipyrromethane (3f). Yellow solid, mp $159{ }^{\circ} \mathrm{C}$ (lit. ${ }^{11} \mathrm{mp} 159-160{ }^{\circ} \mathrm{C}$, lit. yield ${ }^{11} 56 \%$ ). ${ }^{1} \mathrm{H}$ NMR (300 MHz, $\left.\mathrm{CDCl}_{3}\right): 5.58(\mathrm{~s}, 1 \mathrm{H}), 5.87(\mathrm{~s}, 2 \mathrm{H}), 6.17(\mathrm{dd}, J 4.4 \mathrm{~Hz}, 2 \mathrm{H}), 6.75$ (d, J 4.8 Hz, 2H), 7.37-8.02 (m, 4H), 8.17 (brs, 2H, NH). MS m/z (\%): $267\left(\mathrm{M}^{+}, 100\right), 145\left(\mathrm{M}^{+}-\right.$ $\mathrm{C}_{6} \mathrm{H}_{4} \mathrm{NO}_{2}, 80$ ) calculated for $\mathrm{C}_{15} \mathrm{H}_{13} \mathrm{~N}_{3} \mathrm{O}_{2}$.

meso-Tolyldipyrromethane (3g). Buff solid, mp $112{ }^{\circ} \mathrm{C}$ (lit. ${ }^{11} \mathrm{mp} 114{ }^{\circ} \mathrm{C}$, lit. yield ${ }^{11} 33 \%$ ), ${ }^{1} \mathrm{H}$ NMR (300 MHz, $\left.\mathrm{CDCl}_{3}\right): 2.35\left(\mathrm{~s}, 3 \mathrm{H}, \mathrm{CH}_{3}\right), 5.44$ (s, 1H), 5.92 (s, 2H), 6.15 (dd, J 4.2 Hz, 2H), $6.83(\mathrm{~d}, J 4.8 \mathrm{~Hz}, 2 \mathrm{H}), 7.05-7.16(\mathrm{~m}, 4 \mathrm{H}), 7.91$ (brs, 2H, NH). MS $m / z(\%): 236\left(\mathrm{M}^{+}, 15\right), 221$ $\left(\mathrm{M}^{+}-\mathrm{CH}_{3}, 3\right)$ calculated for $\mathrm{C}_{16} \mathrm{H}_{16} \mathrm{~N}_{2}$.

meso-Mesityldipyrromethane (3h). Pale yellow solid. mp $170{ }^{\circ} \mathrm{C}$ (lit, ${ }^{11} \mathrm{mp} 170-171{ }^{\circ} \mathrm{C}$, lit. yield ${ }^{11} 27 \%$ ), ${ }^{1} \mathrm{H}$ NMR (300 MHz, $\left.\mathrm{CDCl}_{3}\right): 2.04$ (s, 6H, $\left.\mathrm{CH}_{3}\right), 2.27\left(\mathrm{~s}, 3 \mathrm{H}, \mathrm{CH}_{3}\right), 5.92(\mathrm{~s}, 1 \mathrm{H}), 6.03$ 
(dd, J 2.6 Hz, 2H), 6.17 (m, 2H), 6.65 (m, 2H), 6.86 (s, 2H), 7.95 (brs, 2H, NH). MS m/z (\%): 264 $\left(\mathrm{M}^{+}, 15\right)$ calculated for $\mathrm{C}_{18} \mathrm{H}_{20} \mathrm{~N}_{2}$.

meso-(4-Methoxyphenyl)dipyrromethane (3i). Brownish white crystals, $\mathrm{mp} 99{ }^{\circ} \mathrm{C}\left(\right.$ lit $^{11} \mathrm{mp}$ $99{ }^{\circ} \mathrm{C}$, lit. yield $\left.{ }^{11} 68 \%\right)$. ${ }^{1} \mathrm{H}$ NMR $\left(300 \mathrm{MHz}, \mathrm{CDCl}_{3}\right): 3.78\left(\mathrm{~s}, 3 \mathrm{H}, \mathrm{OCH}_{3}\right), 5.41(\mathrm{~s}, 1 \mathrm{H}), 5.90$ (s, 2H), $6.13(\mathrm{dd}, J 2.9 \mathrm{~Hz}, 2 \mathrm{H}), 6.82(\mathrm{~d}, J 8.6 \mathrm{~Hz}, 2 \mathrm{H}), 7.10$ (dd, J 8.6 Hz, 4H), 7.89 (brs, 2H, NH). MS $m / z(\%): 252\left(\mathrm{M}^{+}, 94\right), 145\left(\mathrm{M}^{+}-\mathrm{C}_{7} \mathrm{H}_{7} \mathrm{O}, 100\right)$ calculated for $\mathrm{C}_{16} \mathrm{H}_{16} \mathrm{~N}_{2} \mathrm{O}$.

meso-(4-Pyridyl)dipyrromethane (3j). Light brown solid, mp $140{ }^{\circ} \mathrm{C}$ (lit. ${ }^{14} \mathrm{mp} 140{ }^{\circ} \mathrm{C}$, lit. yield ${ }^{14}$ $58 \%) .{ }^{1} \mathrm{H}$ NMR (300 MHz, $\left.\mathrm{CDCl}_{3}\right): 5.44$ (s, 1H), 5.89 (s, 2H), 6.60 (dd, J 4.6 Hz, 2H), 7.09 (d, $J$ $8.2 \mathrm{~Hz}, 2 \mathrm{H}), 8.41(\mathrm{~m}, 2 \mathrm{H}), 10.62(\mathrm{~s}, 2 \mathrm{H}, \mathrm{NH}) . \mathrm{MS} m / z(\%): 223\left(\mathrm{M}^{+}, 100\right)$ calculated for $\mathrm{C}_{14} \mathrm{H}_{13} \mathrm{~N}_{3}$. meso-Acetyldipyrromethane (3k). Viscous brown liquid. $\left.{ }^{1} \mathrm{H} \mathrm{NMR} \mathrm{(300} \mathrm{MHz,} \mathrm{CDCl} 3\right): 2.26$ (s, $\left.3 \mathrm{H}, \mathrm{CH}_{3}\right), 5.14(\mathrm{~s}, 1 \mathrm{H}), 6.15(\mathrm{~d}, J 2.4 \mathrm{~Hz}, 2 \mathrm{H}), 6.16(\mathrm{~d}, J 2.4 \mathrm{~Hz}, 2 \mathrm{H}), 6.7(\mathrm{~d}, J 0.3 \mathrm{~Hz}, 2 \mathrm{H}), 8.46$ (brs, $2 \mathrm{H}, \mathrm{NH})$. HRMS $m / z(\%): 189.1020\left(\mathrm{M}+\mathrm{H}^{+}, 100\right)$ calculated for $\mathrm{C}_{11} \mathrm{H}_{13} \mathrm{~N}_{2} \mathrm{O}$ 189.1028. The spectral data of compound 3k are reproduced in the Supplementary Data File.

\section{Acknowledgements}

The authors gratefully acknowledge financial assistance from the Council of Scientific and Industrial Research, New Delhi, India. We also thank University Science Instrumentation center (USIC), University of Delhi, India for spectral analysis of compounds.

\section{Supplementary Data}

The ${ }^{1} \mathrm{H}$ and ${ }^{13} \mathrm{C}$ NMR spectra, and the HRMS trace in the region of $\mathrm{M}^{+}$, of compound $\mathbf{3 k}$, are contained in the Supplementary File.

\section{References}

1. Ptaszek, M.; McDowell, B. E.; Lindsey, J. S. J. Org. Chem. 2006, 71, 4328-4331. https://doi.org/10.1021/jo060119b

2. Abada, Z.; Ferrie, L.; Akagah, B.; Lormier, A. T.; Figadere, B. Tetrahedron Lett. 2011, 52, 3175-3178. https://doi.org/10.1016/j.tetlet.2011.04.028

3. Kim, H. J.; Dogutan, D. K.; Ptaszek, M.; Lindsey, J. S. Tetrahedron 2007, 63, 37-55. https://doi.org/10.1016/j.tet.2006.10.027

4. Golf, H. R. A.; Reissig, H. U.; Wiehe, A. J. Org. Chem. 2015, 80, 5133-5143. https://doi.org/10.1021/acs.joc.5b00528 
5. Eshghi, H.; Rahimizadeh, M.; Attaran, N.; Bakavoli, M. J. Iran Chem. Soc. 2013, 10, 11511157. https://doi.org/10.1007/s13738-013-0253-1

6. Nishino, N.; Wagner, R. W.; Lindsey, J. S. J. Org. Chem. 1996, 61, 7534-7544. https://doi.org/10.1021/jo9611576

7. Milgrom, L. R.; Yahioglu, G. Tet. Lett. 1996, 37, 4069-4072. https://doi.org/10.1016/0040-4039(96)00735-6

8. Lee, C. H.; Lindsey, J. S. Tetrahedron 1994, 50, 11427-11440. https://doi.org/10.1016/S0040-4020(01)89282-6

9. Mizutani, T.; Ema, T.; Tomita T.; Kuroda. Y.; Ogoshi. H. J. Am. Chem. Soc. 1994, 116, 4240-4250. https://doi.org/10.1021/ja00089a013

10. Vigmond, S. J.; Chang, M. C.; Kallury, K. M. R.; Thompson, M. Tet. Lett. 1994, 35, 2455-2458. https://doi.org/10.1016/S0040-4039(00)77142-5

11. Littler, B. J.; Miller, M. A.; Hung, C.; Wagner, R. W.; O’Shea, D. F.; Boyle, P. D.; Lindsey, J. S. J. Org. Chem. 1999, 64, 1391-1396. https://doi.org/10.1021/jo982015+

12. Zhao, H.; Liao, J.; Ning, J.; Xie, Y.; Cao, Y.; Chen, L.; Yang, D.; Wang, B. Adv. Synth. Catal. 2010, 352, 3083-3088. https://doi.org/10.1002/adsc.201000336

13. Temelli, B.; Unaleroglu, C. Tetrahedron 2006, 62, 10130-10135. https://doi.org/10.1016/j.tet.2006.08.047

14. Gryko, D.; Lindsey, J. S. J. Org. Chem. 2000, 65, 2249-2252. https://doi.org/10.1021/jo9918100

15. Zhang, Y.; Liang, J.; Shang.Z.-c. Chin. J. Chem. 2010, 28, 259-262. https://doi.org/10.1002/cjoc.201090063

16. Ludwig, R. Angew. Chem. Int. Ed. 2001, 40, 1808-1827. https://doi.org/10.1002/1521-3773(20010518)40:10<1808::AID-ANIE1808>3.0.CO;2-1

17. Sobral, A. J. F. N. J. Chem. Educ. 2006, 83, 1665-1666. https://doi.org/10.1021/ed083p1665

18. Kral, V. Vasek, P.; Dolensky, B. Collect. Czech. Chem. Commun. 2004, 69, 1126-1136.

19. Chauhan, S. M. S.; Singh, R.; Gulati, A. Indian J. Heterocycl. Chem. 2000, 9, 231-232.

20. Chauhan, S. M. S.; Garg, B.; Bisht, T. Molecules 2007, 12, 2458-2466. https://doi.org/10.3390/12112458

21. Chauhan, S. M. S.; Rawat, A. K. Der Pharma Chemica 2014, 6, 316-322.

22. Chauhan, S. M. S.; Mishra, S. Molecules 2011, 16, 7256-7266. https://doi.org/10.3390/molecules16097256

23. Naik, R.; Joshi, P.; Kaiwar, S. P.; Deshpande, R. K. Tetrahedron 2003, 59, 2207-2213. https://doi.org/10.1016/S0040-4020(03)00245-X 
24. Senapak, W.; Saeeng, R.; Jaratjaroonphong, J.; Kasemsuk, T.; Siroin, U. Org. Biomol. Chem. 2016, 14, 1302-1310.

https://doi.org/10.1039/C5OB01953B

25. Pereira, N. A. M.; Pinho e Melo, T. M. V. D. Organic Preparations and procedures International, 2014, 46, 183-213.

https://doi.org/10.1080/00304948.2014.903140

26. Yadav, J. S.; Gupta, M. K.; Jain, R.; Yadav, N. N.; Reddy, B. V. S. Monatsh Chem. 2010, 141, 1001-1004.

https://doi.org/10.1007/s00706-010-0355-8

27. Jaberi, Z. K.; Keshavarzi, M. Chin. Chem. Lett. 2010, 21, 547-549.

https://doi.org/10.1016/j.cclet.2010.01.014

28. Stahlberg, T.; Rodriguez, S. R.; Fristrup, P.; Riisager, A. Chem. Eur. J. 2011, 17, 14561464.

https://doi.org/10.1002/chem.201002171

29. Rostami, A.; Akradi, J.; Jangi, F. A. J. Brazilian Chem. Soc. 2010, 21, 1587-1592. https://doi.org/10.1590/S0103-50532010000800026

30. Garimallaprabhakaran, A.; Harmata, M. Synlett 2011, 361-364.

31. Nath, J.; Chaudhari, M. K. Green Chem. Lett. Rev. 2008, 1, 223-230. https://doi.org/10.1080/17518250902758887

32. Chaudhuri, M. K.; Hussain, S.; Kantam, M. L.; Neelima, B. Tet. Lett. 2005, 46, 83298331.

https://doi.org/10.1016/j.tetlet.2005.09.167

33. Tu, S.; Fang, F.; Miao, C.; Jiang, H.; Feng, Y.; Shi, D.; Wang, X. Tet. Lett. 2003, 44, 6153-6155.

https://doi.org/10.1016/S0040-4039(03)01466-7

34. Chaudhuri, M. K.; Hussain, S. J. Mol. Catal. A: Chem. 2007, 269, 214-217. https://doi.org/10.1016/j.molcata.2007.01.014

35. Rostami, A.; Akradi, Z. Tetrahedron Lett. 2010, 51, 3501-3503. https://doi.org/10.1016/j.tetlet.2010.04.103

36. Tang, P.; Krause, H.; Fürstner, A. Org. Synth. 2005, 81, 262-272. https://doi.org/10.15227/orgsyn.081.0262 